\title{
Title:
}

Acute emphysematous cholecystitis due to clostridium perfringens: rare entity requiring aggressive and early treatment

Authors:

Julio Santoyo Villalba, Inés Cañas García, Mónica Mogollón González

DOI: $10.17235 /$ reed.2022.8631/2022

Link: PubMed (Epub ahead of print)

Please cite this article as:

Santoyo Villalba Julio, Cañas García Inés, Mogollón González Mónica. Acute emphysematous cholecystitis due to clostridium perfringens: rare entity requiring aggressive and early treatment. Rev Esp Enferm Dig 2022. doi:

10.17235/reed.2022.8631/2022.

This is a PDF file of an unedited manuscript that has been accepted for publication. As a service to our customers we are providing this early version of the manuscript. The manuscript will undergo copyediting, typesetting, and review of the resulting proof before it is published in its final form. Please note that during the production process errors may be discovered which could affect the content, and all legal disclaimers that apply to the journal pertain. 


\section{Acute emphysematous cholecystitis due to clostridium perfringens: rare entity requiring aggressive and early treatment}

1. Julio Santoyo Villalba. General and Digestive Surgery Unit. Virgen de las Nieves University Hospital (Granada)

2. Inés Cañas García. General and Digestive Surgery Unit. San Cecilio University Hospital (Granada)

3. Mónica Mogollón González. General and Digestive Surgery Unit. Virgen de las Nieves University Hospital (Granada)

\section{Corresponding author:}

Name: Julio Santoyo Villalba

Email: jsantoyovil@gmail.com

Keywords: Acute emphysematous cholecystitis. Cholecystectomy. Clostridium perfringens.

\section{Letter to the Editor}

Acute emphysematous cholecystitis is a rare entity ( $1 \%$ of acute cholecystitis) characterized by the presence of gas in the gallbladder lumen, gallbladder wall or even in the bile duct (pneumobilia). It is associated with arteriosclerosis, embolic events, and diabetes mellitus ${ }^{1}$ and as in other similar pathologies such as emphysematous gastritis or emphysematous pancreatitis ${ }^{2,3}$ the most frequently isolated microorganisms are Streptococcus pyogenes, Escherichia coli and Staphylococcus aureus, although others such as Proteus vulgaris, Pseudomonas aeruginosa, Clostridium perfringens and Klebsiella pneumoniae have also been found. This disease 
has a significantly higher morbidity and mortality than the rest of acute cholecystitis and therefore requires early diagnosis and rapid and aggressive treatment.

The case of an 82-year-old patient with a history of hypertension, dyslipidemia and morbid obesity who consults the Emergency Department due to abdominal pain in the right hypochondrium and vomiting of 72 hours of evolution is described. On physical examination, a painful abdomen in the epigastrium and right hypochondrium with a positive Murphy's sign is evident. Blood analysis is performed showing marked elevation of acute phase reactants (CRP 365, Procalcitonin 1.86 and Leukocytosis $19,600)$. The study is completed with radiography, ultrasound, and CT of the abdomen (Figure 1) that reports acute emphysematous cholecystitis with marked inflammatory changes near the gallbladder.

Broad-spectrum antibiotic therapy is initiated with Piperacillin-Tazobactam 4gr every 6 hours and urgent laparoscopic cholecystectomy is performed, demonstrating a completely gangrenous gallbladder. The postoperative period is satisfactory, being discharged on the fifth day after surgery. In the culture of bile Clostridium perfringens susceptible to most antibiotics grows, completing the patient the antibiotic cycle at home with Amoxicillin-Clavulanic.

Treatment of emphysematous cholecystitis should be aggressive, including intensive fluid therapy, broad-spectrum antibiotic therapy covering aerobic and anaerobic germs, and if the patient's situation allows it, urgent cholecystectomy should be performed.

\section{Bibliography}

1. Koole SN, Lohman BG, van Unen JM. Emphysematous cholecystitis due to Clostridium perfringens successfully treated by cholecystectomy. Acta Chir Belg. 2016;116(1):54-57.

2. Gil-Díez López-Maroto D, Rodríguez Cuéllar E, Nevado García C et al. Emphysematous esophagitis with gastric perforation. Rev Esp Enferm Dig. 2019;111(11):884-886 
3. Ramírez Esteso F, Domínguez Ferreras E, Domper Bardají $F$ et al. Emphysematous pancreatitis: a rare entity with characteristic radiological findings. Rev Esp Enferm Dig. 2021;113(10):721-722.

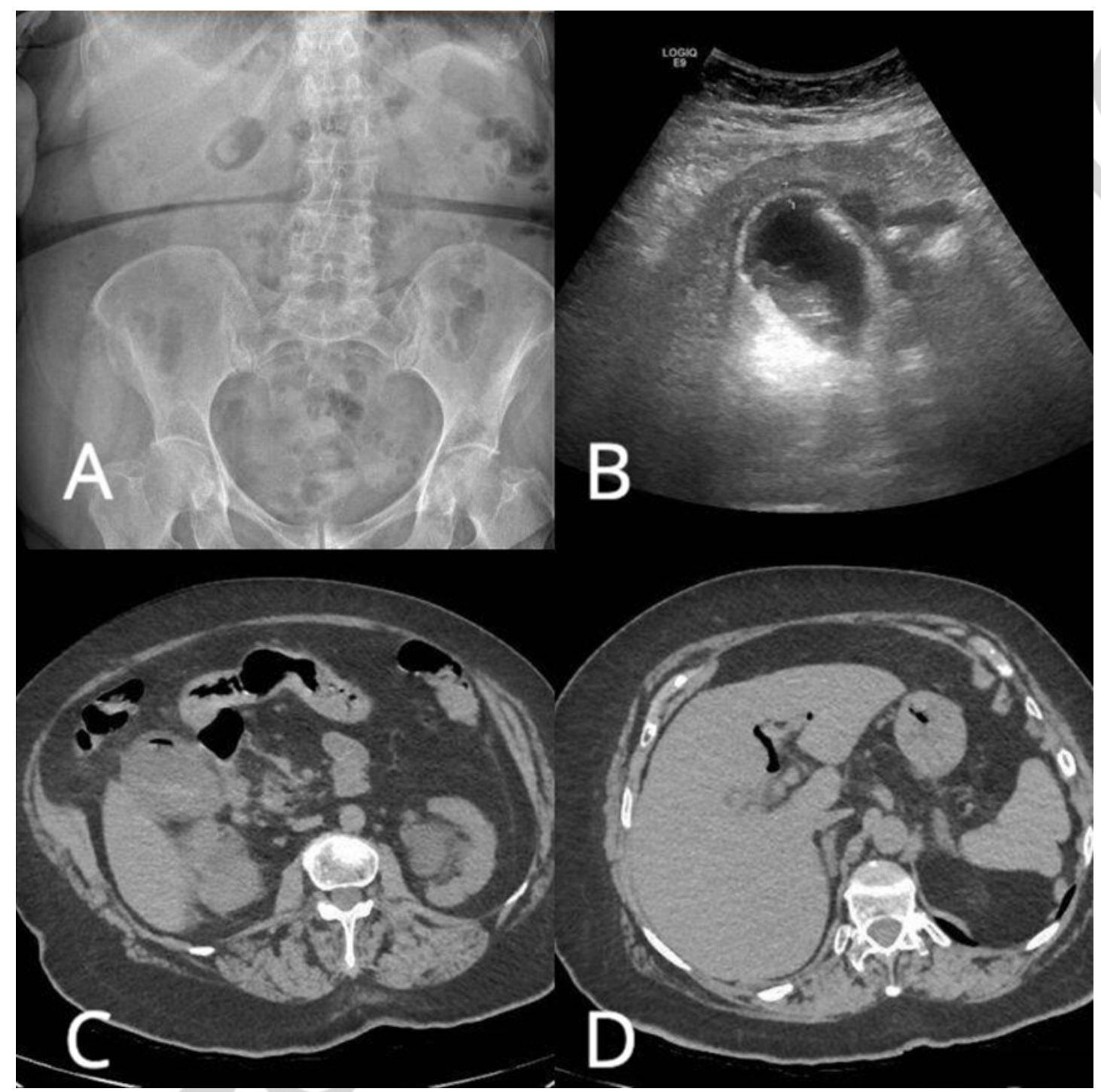

Figure 1. Radiological study. (A) Abdominal X-ray in which gas and gallstone are evident in the gallbladder lumen. (B) Ultrasound of the abdomen showing stratified thickening of gallbladder wall. (C) and (D) CT axial slices where gas in gallbladder wall and pneumobilia in left radicals are evident. 